\title{
Inhibition of 4-aminobutyrate aminotransferase protects against injury-induced osteoarthritis in mice
}

\author{
Jie Shen, ${ }^{1}$ Cuicui Wang, ${ }^{1}$ Jun Ying, ${ }^{1,2,3}$ Taotao Xu, ${ }^{1,2,3}$ Audrey McAlinden, ${ }^{1,4}$ and Regis J. O'Keefe ${ }^{1}$ \\ 'Department of Orthopaedic Surgery, School of Medicine, Washington University in St. Louis, St. Louis, Missouri, USA. \\ ${ }^{2}$ Institute of Orthopaedics and Traumatology, the First Affiliated Hospital of Zhejiang Chinese Medical University, \\ Hangzhou, China. ${ }^{3}$ Zhejiang Chinese Medical University, Hangzhou, China. ${ }^{4}$ Department of Cell Biology and Physiology, \\ School of Medicine, Washington University in St. Louis, St. Louis, Missouri, USA.
}

\begin{abstract}
Recently we demonstrated that ablation of the DNA methyltransferase enzyme, Dnmt3b, resulted in catabolism and progression of osteoarthritis (OA) in murine articular cartilage through a mechanism involving increased mitochondrial respiration. In this study, we identify 4-aminobutyrate aminotransferase (Abat) as a downstream target of Dnmt3b. Abat is an enzyme that metabolizes $\gamma$-aminobutyric acid to succinate, a key intermediate in the tricarboxylic acid cycle. We show that Dnmt3b binds to the Abat promoter, increases methylation of a conserved $\mathrm{CpC}$ sequence just upstream of the transcriptional start site, and inhibits Abat expression. Dnmt3b deletion in articular chondrocytes results in reduced methylation of the $C p C$ sequence in the Abat promoter, which subsequently increases expression of Abat. Increased Abat expression in chondrocytes leads to enhanced mitochondrial respiration and elevated expression of catabolic genes. Overexpression of Abat in murine knee joints via lentiviral injection results in accelerated cartilage degradation following surgical induction of OA. In contrast, lentiviral-based knockdown of Abat attenuates the expression of IL-1ß-induced catabolic genes in primary murine articular chondrocytes in vitro and also protects against murine articular cartilage degradation in vivo. Strikingly, treatment with the FDA-approved small-molecule Abat inhibitor, vigabatrin, significantly prevents the development of injury-induced OA in mice. In summary, these studies establish Abat as an important new target for therapies to prevent $\mathrm{OA}$.
\end{abstract}

Conflict of interest: The authors have declared that no conflict of interest exists.

Copyright: () 2019, American Society for Clinical Investigation.

Submitted: March 4, 2019

Accepted: August 8, 2019

Published: September १९, 2019.

Reference information: /CI Insight. 2019;4(18):e128568.

https://doi.org/10.1172/jci.

insight.128568.

\section{Introduction}

Osteoarthritis (OA), the most common degenerative joint disorder, places an enormous financial burden on the health care system and results in marked disability in the population (1-4). By the year 2030, nearly 67 million people will be suffering from OA across the United States (5). Currently, knee and hip OA is diagnosed by radiographs showing significant articular cartilage loss, joint space narrowing, subchondral bone sclerosis, osteophytes, extremity deformity, and severe inflammation. There are no effective disease-modifying treatments for OA other than pain management and end-stage surgical interventions (6-8). Thus, there is an urgent unmet clinical need to develop mechanistically based therapeutic strategies to treat this disease.

Although OA is a disease affecting the whole joint, articular cartilage destruction is considered the primary pathological change related to symptoms and disability (9). Numerous GWAS have examined gene sequence changes that might predispose individuals to develop OA. Although several gene mutations and single nucleotide polymorphisms have been identified, alterations in DNA base sequences account for the minority of cases of osteoarthritis across the population (10-15). More recently, the role of epigenetics in the modulation of gene expression and in the pathogenesis of human diseases has been recognized. Epigenetic changes involve alterations in the structure of DNA rather than the genetic sequence. The 3 main categories of epigenetic regulation are histone modification (methylation, acetylation, etc.), DNA methylation, and small noncoding RNAs. Epigenetic events that influence gene expression are known to be controlled by aging, disease, and environmental factors, including obesity, smoking, and injury (16-19).

We recently reported that Dnmt3b, one of the DNA methyltransferases, is highly expressed in healthy murine and human cartilage. Our work further established that Dnmt3b loss of function (LOF) in articular 
chondrocytes leads to OA progression, at least in part, because of accelerated tricarboxylic acid (TCA) cycle and elevated mitochondrial respiration (20). Interestingly, increasing evidence implicates an association between increased mitochondrial respiration and cartilage catabolism leading to OA. Specifically, chondrocytes with an OA-like catabolic phenotype transition to oxidative phosphorylation to produce ATP, in contrast with healthy articular chondrocytes that rely on glycolysis to maintain a homeostatic state (21-25). However, the molecular mechanisms and targets involved in the shift toward oxidative phosphorylation in chondrocytes in OA are not known. In the current study, the integrative analysis of RNA-Seq and DNA Methyl-Seq (whole genome bisulfite DNA methylation sequencing) data from Dnmt3b LOF chondrocytes reveals that the enzyme 4-aminobutyrate aminotransferase (Abat) not only is upregulated in OA but also is one of the downstream targets of Dnmt3b. Abat is a critical enzyme responsible for catabolism of $\gamma$-aminobutyric acid (GABA) to succinate (26), an important metabolite in the TCA cycle. Elevated succinate levels enhance the TCA cycle and lead to increased mitochondrial respiration (27).

Human patients with Abat deficiency are diagnosed with cognitive disorders and seizures (27-29). Multiple pharmacological compounds have been developed to target Abat-related pathways to treat these neurological conditions (30-32). In the current study, we examine the role of Abat in the pathogenesis of $\mathrm{OA}$ and explore the potential of vigabatrin, an FDA-approved small-molecule Abat inhibitor, to prevent the development of OA in mice following joint injury. Our data indicate that both Abat and mitochondrial metabolism by oxidative phosphorylation are novel targets for the prevention and treatment of OA.

\section{Results}

Abat is the downstream target of Dnmt3b in chondrocytes. Our recent findings demonstrated that Dnmt $3 b$ gene deletion in chondrocytes leads to a catabolic phenotype in vitro and results in spontaneous articular cartilage degeneration in the knee joints of mice (20). RNA-Seq and DNA Methyl-Seq analyses showed enrichment of differentially expressed genes involved in cellular metabolic processes in articular chondrocytes with Dnmt3b gene deletion. In particular, TCA cycle metabolites and mitochondrial respiration were elevated in Dnmt3b LOF chondrocytes (20). Given that Dnmt3b broadly targets the whole genome and thus may not be an effective therapeutic target, we further examined downstream genes showing both differential expression and methylation patterns in the promoter regions of articular chondrocytes following Dnmt3b LOF. In the current study, we report 44 gene candidates that not only displayed altered expression levels in Dnmt $3 \mathrm{~b}$ LOF chondrocytes but whose promoters also contained alterations in levels of DNA methylation (see Supplemental Table 1; supplemental material available online with this article; https://doi.org/10.1172/jci. insight.128568DS1). Two-thirds of the candidate genes contained hypomethylated promoter CpG regions, including those known to be altered in OA, such as Wif1 (33), Mmp3, and Adam12. Among the remaining genes, we selected $A b a t$, the gene encoding the enzyme Abat for further study because of its potential role in regulating metabolism, and it has not been studied before in the context of cartilage biology to our knowledge. Abat functions to catalyze GABA into succinic semialdehyde, the latter of which can be further oxidized to succinate and enter the TCA cycle (34). We first supported our sequencing data by direct analysis of the Abat promoter and confirmed that Dnmt3b LOF resulted in reduced methylation in this region (Figure 1A) and established that the decrease in methylation likely results in upregulation of $A b a t$ expression in articular chondrocytes (Figure 1B). Our previous observation of increased succinate levels in Dnmt3b LOF chondrocytes (20) suggested that the increase in Abat expression may have functional significance. We next addressed whether Dnmt3b is directly involved in the transcriptional regulation of Abat. A 342-bp CpG island was identified in the proximal promoter of Abat, and ChIP assays showed that Dnmt3b binds specifically to this conserved CpG island of Abat in ATDC5 cells (Figure 1C). To determine the functional role of Dnmt3b binding, we overexpressed Dnmt3b in chondrocytes, and, as expected, expression of Abat decreased as a result (Figure 1D). We previously showed that DNMT3B is decreased in human OA articular cartilage (20), and we further show here that this decrease is associated with a significant increase in $A B A T$ expression in OA cartilage from human patients with OA (Figure 1E). Chondrocytes were isolated from patients with OA and treated with IL-1 $\beta$, which has been shown to increase cartilage catabolism and reduce the expression of Dnmt3b in articular chondrocytes (20). Treatment of human chondrocytes with IL-1 $\beta$ resulted in upregulation of $A B A T$ mRNA expression (Figure $1 \mathrm{~F}$ ). In addition, a robust increase in Abat mRNA and protein expression was also found in murine cartilage tissue 4 weeks after meniscal ligament injury (MLI) surgery (Figure 1G). Altogether, these results show that the decrease in Dnmt3b expression observed in OA results in reduced methylation of the Abat promoter and a concomitant increase 

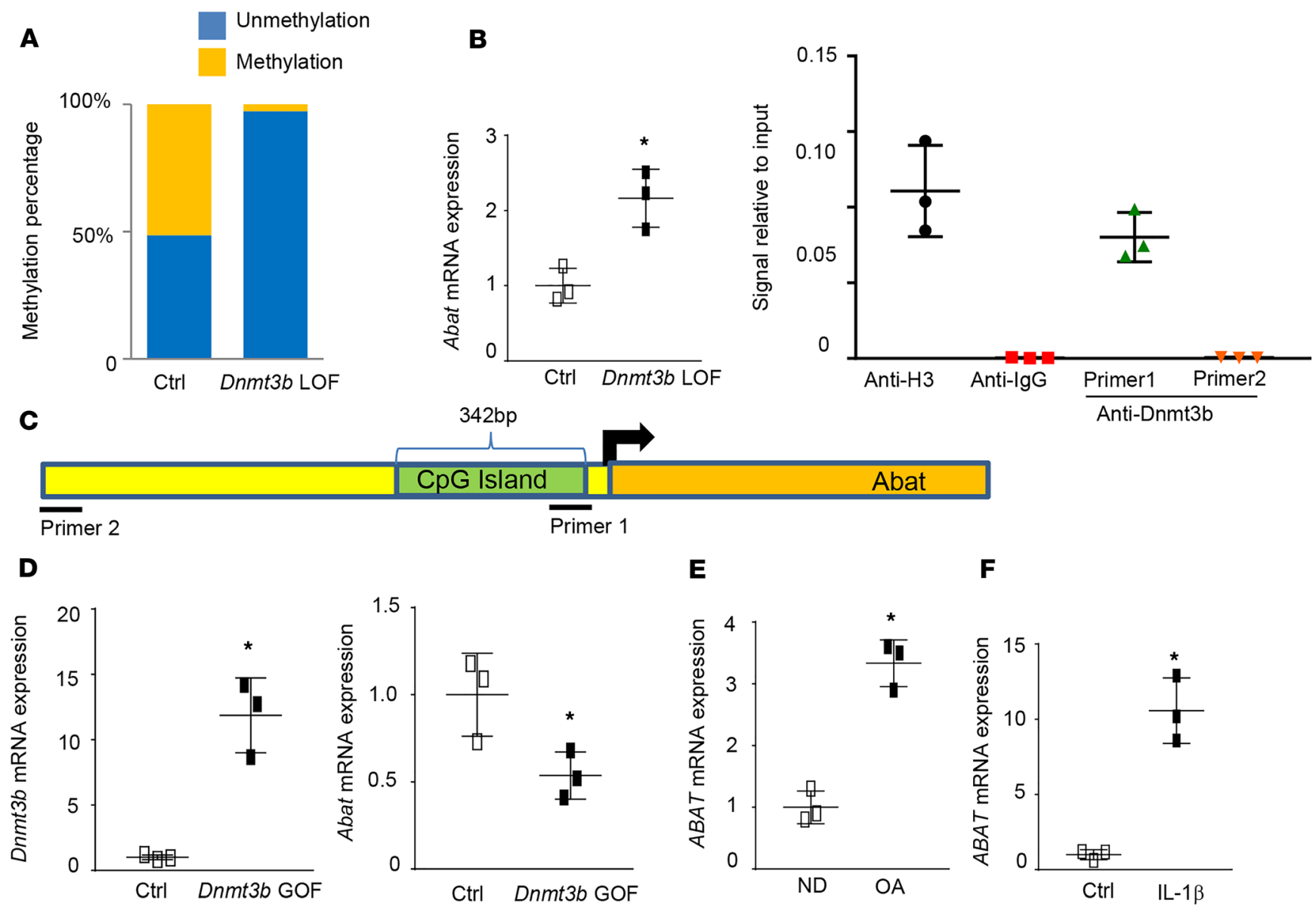

CpG Island

Abat

Primer 1
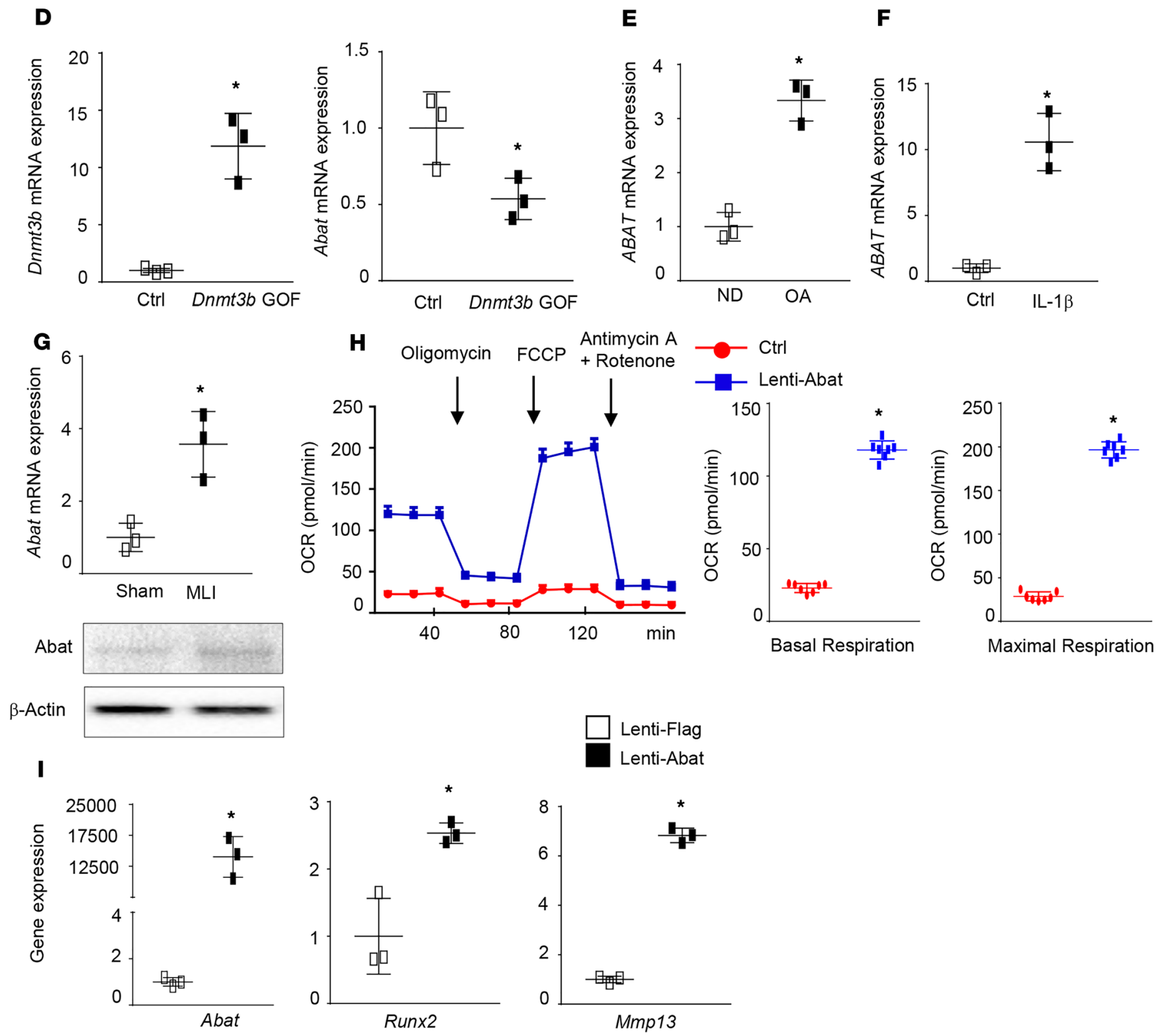
Figure 1. Abat is a target of Dnmt3b, and Abat overexpression induces chondrocyte hypertrophy. Dnmt3b LOF leads to DNA hypomethylation (A) and increased Abat expression (B) in murine chondrocytes $(n=3)$. (C) Pull-down assay of genomic DNA with a Dnmt3b antibody (ChIP assay) shows interaction of Dnmt3b with its binding site in the Abat promoter region by quantitative PCR (qPCR) using specific primers ( $n=3$ ). (D) Real-time qPCR analysis of Dnmt3b and Abat in articular chondrocytes transduced with either lentivirus Dnmt3b (lenti-Dnmt3b) or control virus. (E) Human OA chondrocytes exhibit low ABAT expression compared with nondiseased (ND) chondrocytes $(n=3)$, and (F) induction of human chondrocytes ( $n=3$ ) with IL-1 $\beta$ results in decreased expression of $A B A T$ mRNA. (G) Abat mRNA and protein expression is decreased in MLI cartilage compared with sham surgery cartilage in gene and protein levels ( $n=5$ ) 2 weeks after MLI surgery. (H) Mitochondrial respiration was measured by the Seahorse XF Extracellular Flux Analyzer. Basal respiration and maximal respiration, as measured by the oxygen consumption rate (OCR), are shown $(n=8)$. (I) Real-time qPCR analyses of gene expression from articular chondrocytes transduced with either lenti-Abat or control virus $(n=3)$. ${ }^{*} P<0.05$ by 2-tailed Student's $t$ test.

in Abat gene and protein expression.

Abat overexpression leads to chondrocyte hypertrophy in vitro and accelerated $O A$ progression in mice. To establish the potential role of Abat as a regulator of cellular metabolism and homeostasis, we used lentivirus to overexpress Abat in primary murine articular chondrocytes. Similar to Dnmt3b LOF, lenti-Abat infection enhanced mitochondrial respiration, as reflected by increased basal and maximal oxygen consumption rate (OCR) in articular chondrocytes (Figure 1H). Moreover, overexpression of Abat in articular chondrocytes induced catabolic gene expression, including Runx2 and Mmp13, which is consistent with chondrocyte hypertrophy (Figure 1I). We then directly injected lenti-Abat into murine knee joints immediately following MLI surgery to determine whether Abat overexpression could accelerate cartilage degeneration in mice. Intra-knee injection resulted in successful transduction of lentivirus into cells of the knee joint in vivo (Supplemental Figure 1, A and C), and about $40 \%$ of articular chondrocytes were infected by lenti-Flag and Lenti-Abat virus (Supplemental Figure 1B). At 4 weeks following MLI, articular cartilage in control animals transduced with lenti-Flag remained intact, with no structural changes observed in the subchondral bone. In contrast, the overexpression of Abat in murine knee joints resulted in apparent damage of cartilage tissue 4 weeks following MLI surgery as shown by reduced proteoglycan staining and loss of cartilage tissue (Figure 2A). Additionally, although subchondral bone structure, including bone density and trabecular properties, was similar to control mice (Figure 2B and Supplemental Figure 2, A and B), 6 out of 7 mouse knee joints injected with lenti-Abat developed osteophytes adjacent to the medial tibial plateau (Supplemental Figure 2C), consistent with an OA phenotype. The accelerated OA progression in lenti-Abat-infected knees was further shown by an increase in the OARSI score and decrease in the cartilage area compared with mice infected with lenti-Flag virus (Figure 2, C and D). To examine specific changes in extracellular matrix, expression of Prg4, Col10A1, and Mmp13 was analyzed in articular cartilage tissue from lenti-Flag- and lenti-Abat-infected knees. Immunostaining data showed markedly reduced Prg4 expression, especially in the superficial layer of lenti-Abat-infected cartilage. In addition, abundant staining for Mmp13 and Col10A1 was detected in the cartilage tissue of lenti-Abat-infected mice, compared with minimal expression levels observed in control mice infected with lenti-Flag virus (Figure 2E). The cartilage change and ectopic bone formation induced by Abat overexpression in murine knee joints suggest disruption of homeostasis toward a more catabolic state and that elevated levels of Abat may contribute to the development or accelerated progression of OA.

Inhibition of Abat in vivo attenuates $O A$ progression. Because Abat overexpression led to a catabolic phenotype in chondrocytes associated with increased mitochondria respiration in vitro and accelerated progression of OA in vivo, we next determined whether Abat inhibition could attenuate these effects. Primary murine articular chondrocyte cultures were infected with either control lenti-GFP or lenti-shAbat virus to suppress Abat expression. As expected, both basal and maximal mitochondrial respiration were suppressed by Abat inhibition (Figure 3A and Supplemental Figure 3). Articular chondrocytes were also treated with IL-1 $\beta$, to induce a catabolic phenotype. Transduction of cells with lenti-shAbat inhibited the increase in Col10a1, Runx2, and Mmp13 expression induced by IL-1 $\beta$ treatment (Figure 3B). To determine whether Abat inhibition could also reduce chondrocyte catabolism in vivo, murine knee joints were injected with lenti-GFP or lenti-shAbat following MLI surgery. Supplemental Figure 4 shows that chondrocytes in articular cartilage of the treated knee joints were successfully transduced as shown by positive GFP expression. The lenti-GFP control mice exhibited advanced osteoarthritic changes with loss of proteoglycan and the presence of cartilage surface fibrillations at 10 weeks after MLI. In contrast, cartilage destruction was attenuated in knee joints infected 
A

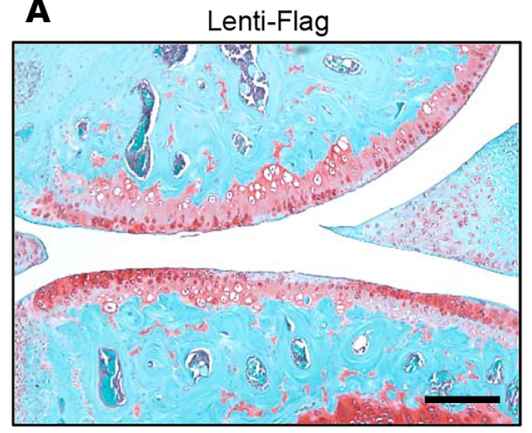

B

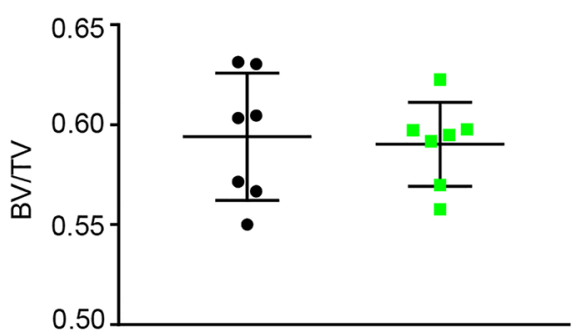

E
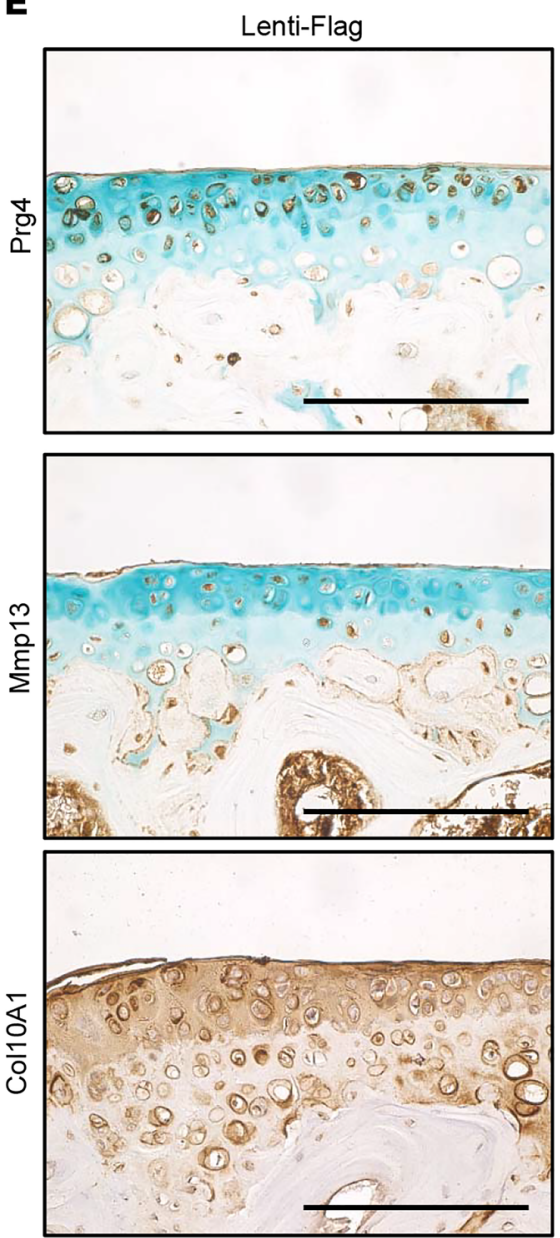

C

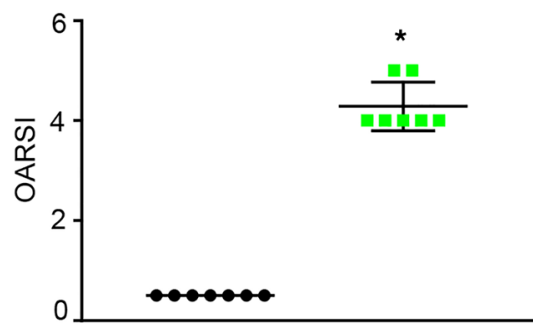

Lenti-Abat

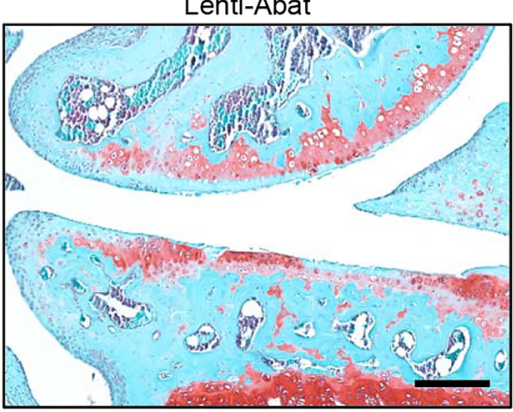

D

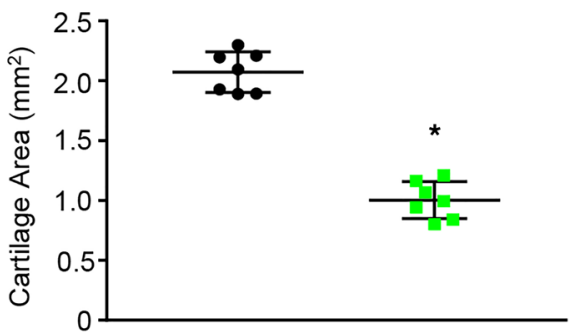

Figure 2. Abat overexpression accelerates $\mathrm{OA}$ progression in mice. MLI or sham surgeries were performed on right knee joints followed by intra-articular injection of lenti-Abat (Abat gain of function [GOF]) or lenti-Flag (control). (A) Representative images of histological sections of control and Abat GOF knee joints at 4 weeks following MLI surgery $(n=7)$. (B) Ratios of bone volume to total volume (BV/TV) of tibial subchondral bone were calculated from the micro-CT images $(n=7)$. Quantification of histological assessment by Osteoarthritis Research Society International (OARSI) scoring (C) and cartilage area (D) $(n=7)$. (E) Immunohistochemical analyses for Prg4, Mmp13, and Col10A1 on knee sections of control and Abat GOF cartilage. ${ }^{*} P<0.05$ by 2 -tailed Student's $t$ test. Scale bars: $50 \mu \mathrm{m}$ 
with lenti-shAbat (Figure 3C). Histological analysis of knee joint tissue sections showed a decrease in OARSI score and preservation of cartilage area because of lenti-shAbat treatment (Figure 3, D and E). Subchondral bone sclerosis is another feature of $\mathrm{OA}$, and we found that lenti-shAbat treatment resulted in lower levels of sclerosis than those observed in lenti-GFP-infected control mice joints (Figure 3F and Supplemental Figure 5, A and B).

Immunohistochemistry was performed to examine the expression of matrix proteins (Prg4, Col10a1) and the catabolic enzyme Mmp13. Preservation of Prg4 expression was apparent in the superficial layer of articular cartilage in the lenti-shAbat-infected knee joints at 10 weeks after MLI, whereas limited Prg4 staining was evident in articular cartilage from control knees. Moreover, the expression of Col10A1 and Mmp13 was also substantially diminished in lenti-shAbatinfected cartilage compared with the abundant expression of Col10A1 and Mmp13 observed in the damaged knee cartilages of control mice infected with the lenti-GFP virus (Figure 3G). Together, these findings strongly suggest that Abat inhibition maintains chondrocyte homeostasis, protects against cartilage degeneration, and attenuates subchondral bone sclerosis in injury-induced OA, implicating Abat as a potential pharmacological target to treat OA.

Vigabatrin treatment protects against injury-induced $O A$ progression in mice. Vigabatrin is an FDA-approved drug that specifically blocks Abat activity and is used clinically to increase GABA levels in neural cells (35-37). Therefore, vigabatrin was added to primary articular chondrocyte cell cultures to determine whether pharmacological inhibition of Abat could maintain cellular homeostasis. To determine the effects of vigabatrin in articular chondrocytes, GABA concentrations were measured in murine primary chondrocyte cultures following vigabatrin treatment. We found that vigabatrin increased GABA levels in articular chondrocytes as expected (Supplemental Figure 6A). Moreover, similar to lenti-shAbat treatment, vigabatrin significantly reduced mitochondrial respiration in chondrocyte cultures (Figure 4A and Supplemental Figure 7), and importantly, vigabatrin inhibited IL-1 $\beta$ mediated induction of the catabolic genes Runx2, Mmp13, and Col10a1 in articular chondrocytes (Figure 4B). To further investigate whether pharmacological blockage of Abat activity by vigabatrin in vivo could modify disease progression in injury-induced OA, MLI surgery was performed on 10-week-old male mice, and vigabatrin was administered daily through intraperitoneal injection at a concentration of $50 \mathrm{mg} / \mathrm{kg}$ or $200 \mathrm{mg} / \mathrm{kg}$ up to 10 weeks following surgery. The vigabatrin injection regime significantly increased GABA concentrations in brain tissue as expected (Supplemental Figure 6B), confirming the pharmacological activity of vigabatrin in vivo. At the lower dose of $50 \mathrm{mg} / \mathrm{kg}$, vigabatrin did not show a protective effect on articular cartilage destruction based on histological examination (Supplemental Figure 8). Strikingly, administration of vigabatrin at $200 \mathrm{mg} / \mathrm{kg}$ for 6 weeks following MLI surgery completely blocked the development of an injury-induced OA phenotype. The apparent articular cartilage surface fibrillation, overall reduced articular cartilage thickness, and increased subchondral bone sclerosis MLI induced was not observed in the vigabatrin-treated mice. By 10 weeks, MLI-induced cartilage damage was more advanced, with pronounced subchondral bone sclerosis in control mice injected with PBS. In contrast, cartilage tissue integrity was maintained by vigabatrin treatment at $200 \mathrm{mg} / \mathrm{kg}$ and subchondral sclerosis was not observed (Figure 4, C and D, and Supplemental Figure 9). Consistent with histological observations, OARSI scoring showed reduced scores, and cartilage area was also maintained in vigabatrin-treated mice at both 6 and 10 weeks following MLI (Figure 4, E and F), demonstrating that Abat inhibition by vigabatrin blocked the development of OA in mice following MLI surgery. Moreover, in contrast with the joints harvested from PBS-treated mice, vigabatrin treatment preserved Prg4 expression in the superficial layer of cartilage tissue, and minimal expression of Mmp13 and Col10A1 in cartilage tissue was observed (Figure 4G). Together, these data define Abat as a promising, novel target for the prevention of OA.

\section{Discussion}

Previously we showed that the expression of the DNA methyltransferase Dnmt $3 \mathrm{~b}$ is reduced in both murine and human articular cartilage in the context of OA. Through loss- or gain-of-function experiments, we established an important mechanistic role for Dnmt3b in maintaining cartilage homeostasis (20). Because DNA methylation is widespread throughout the genome, multiple downstream targets are likely affected via changes in methylation levels within enhancers, promoters, and intronic and exonic regions in the gene body and in intervening regions of DNA remote from a gene. As a starting point, we focused on genes whose expression was significantly altered by Dnmt3b LOF and that also displayed changes in methylation 


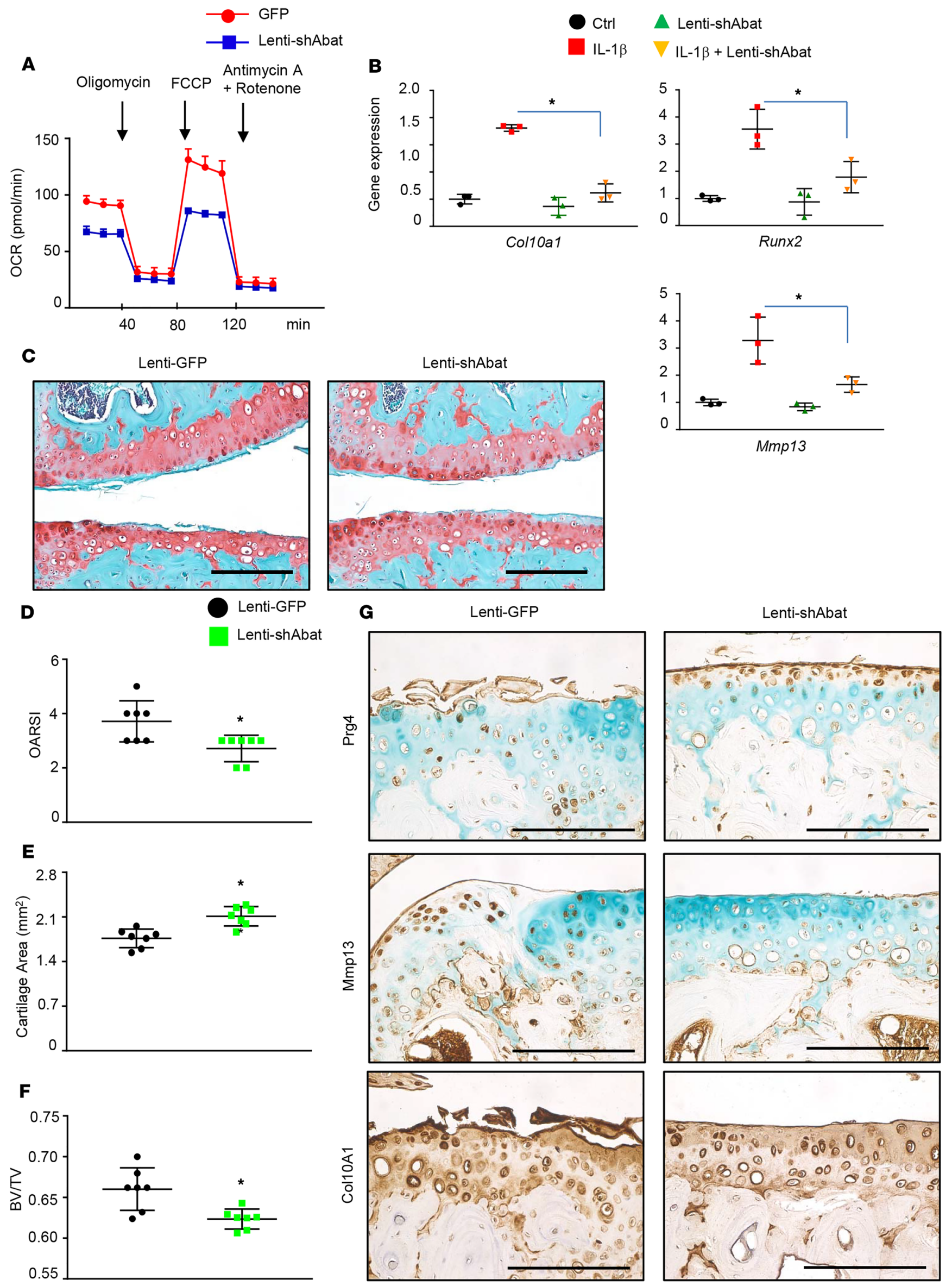


Figure 3. Abat inhibition attenuates chondrocyte hypertrophy and protects cartilage degeneration following surgical induction of $\mathrm{OA}$. (A) Mitochondrial respiration was measured in primary articular chondrocytes treated with lenti-shAbat (Abat LOF) or lenti-GFP (Ctrl) ( $n=8)$. (B) Real-time qPCR analyses of gene expression from articular chondrocytes transduced with either lenti-shAbat (Abat LOF) or control virus following IL-1 $1 \beta$ treatment ( $n=$ 3). (C) Representative images of histological sections from control or Abat LOF knee joints at 10 weeks following MLI surgery $(n=7)$. Quantification of histological assessment by OARSI scoring (D) and cartilage area $(\mathbf{E})(n=7)$. (F) BV/TV ratios of tibial subchondral bone were calculated from the micro-CT images $(n=7)$. (G) Immunohistochemical analyses for Prg4, Mmp13, and Col10A1 on knee sections of control and Abat LOF cartilage. ${ }^{*} P<0.05$ by 2 -tailed Student's $t$ test (A and $\mathbf{D}-\mathbf{F})$ and ANOVA with post hoc test (B). Scale bars: $50 \mu \mathrm{m}$.

patterns in their promoters. Gene ontology analysis of these 44 genes revealed that cell metabolic pathways were a common target. In the current study, we establish one of these genes, Abat, as a novel target involved in the regulation of chondrocyte mitochondria function and the pathogenesis of OA

Abat is an enzyme that catalyzes the conversion of GABA into succinic semialdehyde, which is subsequently oxidized to succinate, a key intermediate in the TCA cycle (34). As we showed that Abat is increased in OA in both mice and humans, we subsequently used a series of gain- and loss-of-function approaches to determine whether Abat is mechanistically involved in the development of OA. We provide in vitro and in vivo evidence that overexpression of Abat leads to chondrocyte hypertrophy, a catabolic phenotype, increased mitochondria respiration, and acceleration of the development of OA in mice. In contrast, Abat inhibition, in vitro and in vivo, maintains cartilage homeostasis, reduces mitochondrial respiration, and attenuates the development of OA following joint injury. Finally, vigabatrin, an irreversible small-molecule inhibitor of Abat, substantially prevented development of OA in mice following MLI surgery.

Recent studies provided increasing evidence that OA is associated with changes in chondrocyte homeostasis that involve alterations in matrix gene expression and mitochondrial function. Specifically, healthy articular chondrocytes maintain a homeostatic state and primarily use glycolysis for energy production (25). In contrast, hypertrophic chondrocytes in the growth plate rely more on oxidative phosphorylation and mitochondrial function to generate ATP (38). Bone morphogenetic protein 2 (BMP2) treatment, which stimulates chondrocyte maturation and hypertrophy in vitro, also stimulates mitochondrial oxidative phosphorylation (25). Although a correlation has been established between oxidative phosphorylation and chondrocyte hypertrophy, it remains unclear whether increased mitochondrial respiration is a primary cause of chondrocyte maturation and is involved in the pathogenesis of OA. To our knowledge, we provide the first genetic evidence in this study that upregulation of cellular metabolism through Abat causes chondrocyte catabolism and leads to OA progression in mice. We show that overexpression of Abat in chondrocytes, as expected, enhances mitochondrial respiration and results in increased expression of catabolic and hypertrophy-related genes. Conversely, Abat inhibition attenuates the expression of catabolic and hypertrophy-related genes in IL-1 $\beta$-treated articular chondrocytes, reduces mitochondrial respiration, and slows the progression of OA in mice following MLI surgery. The current findings with Abat GOF and LOF are consistent with our previous findings showing that suppression of mitochondrial respiration reduced articular chondrocyte hypertrophy (20). Altogether, these findings suggest a connection among Abat, mitochondrial function, cartilage catabolism, and the development of OA. In addition to energy metabolism, Abat regulates mitochondrial nucleoside metabolism and contributes to neurological disorders (27). It will be worthwhile to investigate whether nucleoside metabolism is directly associated with chondrocyte mitochondrial metabolism and OA phenotype in future studies.

In this study, lentiviral vectors were injected into murine knee joints, which would have altered the expression of Abat in synovial tissues, meniscus, and other ligamentous tissues. Although there were no obvious histological changes in these tissues, we did not examine these cells for changes in energy metabolism or gene expression. Because Abat targets an intracellular molecular process, it is unlikely that the molecular events in cartilage were secondary to signals from the synovium, meniscus, or ligament tissues. This is consistent with our finding that similar alterations in the anabolic versus catabolic balance in cartilage were observed in the in vivo and in vitro gain- and loss-of-function experiments. Because mice with Abat gene deletions are not currently available, our results provide the first genetic evidence to our knowledge demonstrating a functional role for Abat in chondrocytes, both in vivo and in vitro. Given its general importance in mitochondrial metabolism, inducible Abat gene deletion mice will be an extremely valuable tool to confirm the specific role of Abat in cartilage and other tissues, including meniscus cells, synovial fibroblasts, and osteoblasts.

Abat is the rate-limiting enzyme that mediates glutamine amino transformation through a GABA 

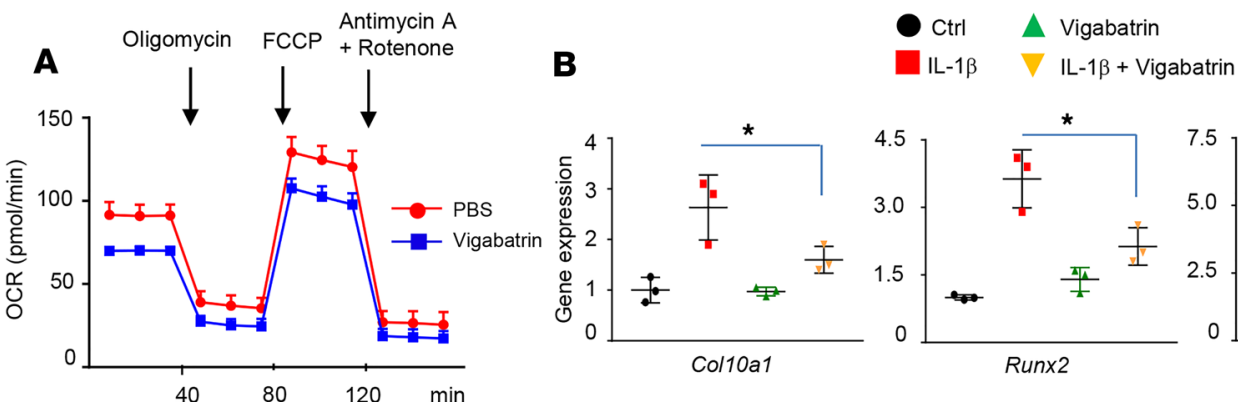

C
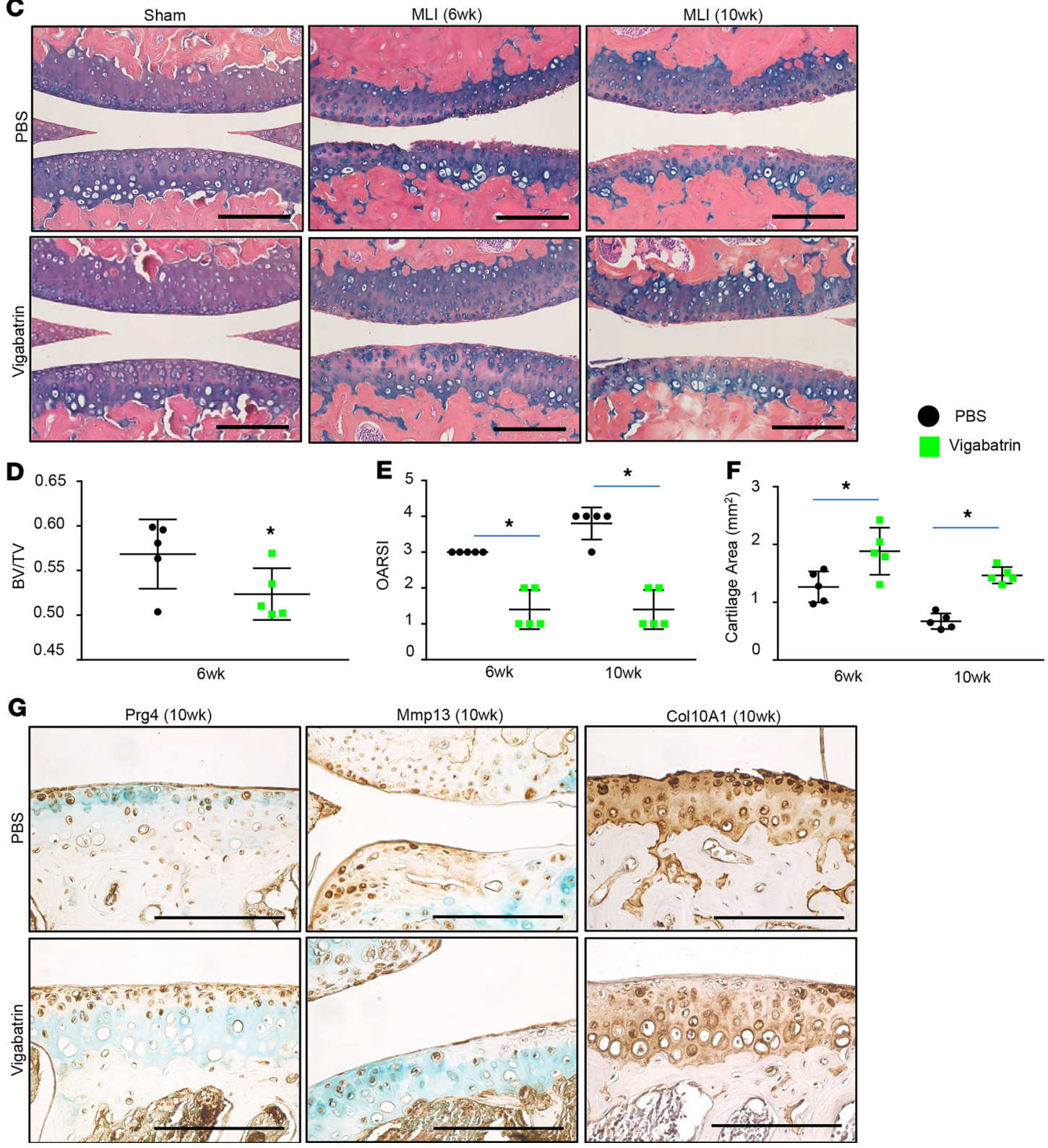

Figure 4. Vigabatrin attenuates chondrocyte hypertrophy and protects against injury-induced OA progression. (A) Mitochondrial respiration was measured in primary articular chondrocytes treated with PBS or vigabatrin $(n=8)$. (B) Real-time qPCR analyses of gene expression from articular chondrocytes pretreated with PBS and vigabatrin $(100 \mu \mathrm{M})$ following IL-1 $1 \beta$ treatment $(n=3)$. (C) Representative images of histological sections of PBS-treated and vigabatrin-treated $(200 \mathrm{mg} / \mathrm{kg}$ ) knee joints at 6 and 10 weeks following MLI surgery $(n=5)$. (D) BV/TV ratios of tibial subchondral bone were calculated from the micro-CT images $(n=7)$. Quantification of histological assessment by OARSI scoring (E) and cartilage area $(\mathbf{F})(n=7)$. (G) Immunohistochemical analyses for Prg4, Mmp13, and Col10A1 on knee sections of PBS- and vigabatrin-treated cartilage 10 weeks after MLI. ${ }^{*} P<0.05$ by 2-tailed Student's $t$ test (A and $\mathbf{D}$ ) and ANOVA with post hoc test (B, E, and F). Scale bars: $50 \mu \mathrm{m}$. 
intermediate to synthesize succinate, which enters the TCA cycle to facilitate energy production through mitochondrial oxidative phosphorylation $(26,34)$. Other sources of succinate for the TCA cycle include glucose and lipid metabolism (39). Our findings indicate that Abat-mediated glutamine metabolism is a potentially important source of succinate in articular chondrocytes because inhibition of Abat had a robust effect on articular chondrocyte energy metabolism and had an important role in the maintenance of chondrocyte homeostasis. However, our findings did not rule out a role for glucose and lipid metabolism as a source of succinate production for use in the TCA cycle.

The most striking finding in this study is that vigabatrin significantly blocked the development of OA in mice with MLI. As an FDA-approved inhibitor of Abat activity that is used to prevent seizures, the kinetics (40-42) have been well studied and documented in different species, including mice, rats, monkeys, and humans (43). We confirmed the inhibitory effect of vigabatrin in brain tissues by measuring an increase in GABA concentrations in harvested brain tissue and found that both $50 \mathrm{mg} / \mathrm{kg}$ and $200 \mathrm{mg} / \mathrm{kg}$ dosages were effective, and neither caused any obvious side effects. However, only mice with $200 \mathrm{mg} / \mathrm{kg}$ systemic vigabatrin injection displayed a protective effect on cartilage tissue in vivo, suggesting that higher systemic concentrations are required to get an enzyme-inhibitory concentration of vigabatrin into articular chondrocytes in the knee joint. Therefore, other methods of administration of vigabatrin or alternative molecular or pharmaceutic approaches to target Abat or other key points of articular chondrocyte metabolism are potential therapeutic approaches. Because pain is a significant clinical symptom in patients with OA, it will be worthwhile to examine the pain response under the vigabatrin treatment as well.

Although lenti-shAbat attenuated the onset of OA in mice with MLI, the effect was partial and much less than was observed with vigabatrin. Fluorescent imaging of lenti-GFP-infected articular cartilage demonstrated that only $40 \%$ of articular chondrocytes were infected. Because Abat works on cell metabolism in a cell-autonomous manner, the ability of lenti-shAbat to block succinate production and decrease mitochondrial oxidative phosphorylation is dependent on the efficacy of the lentiviral infection. In contrast, as an irreversible small-molecule inhibitor that is delivered systemically, vigabatrin appears to have more profound effects.

In addition to its role in energy production, succinate is an intracellular stimulator of inflammatory signaling $(44,45)$. In various cell types, such as dendritic cells, macrophages, and cancer cells, succinate stabilizes HIF-1 $\alpha$ under normoxia conditions and activates IL-1 $\beta$ expression through direct binding to the proximal gene promoter (46-48). In synovial fibroblasts, succinate treatment was found to induce NLRP3-mediated inflammasome activation and increase the intracellular inflammatory responses. However, this inflammation could be attenuated by inhibition of succinate levels in cell cultures (49). Of note, MLI surgeries caused inflammation in synovium tissue, whereas lenti-shAbat and vigabatrin treatment reduced the inflammatory response (Supplemental Figure 1D, Supplemental Figure 4B, and Supplemental Figure 10). Other studies also indicate that vigabatrin inhibits the mammalian target of rapamycin (mTOR) pathway in cell cultures and in murine models (50). The mTOR pathway has been shown as an important regulator in skeletal growth and chondrocyte differentiation in mice (51-53), and inhibition of mTOR has been shown to be associated with enhanced autophagy $(54,55)$. Thus, the ability of vigabatrin to prevent the development of OA following knee injury in mice could be related in part through alternative mechanisms that include inhibition of succinate-mediated inflammation and increased autophagy via mTOR inhibition.

In conclusion, our potentially novel body of research has established Abat as an important downstream target of Dnmt3b and as a critical regulator of cellular metabolism and chondrocyte hypertrophy/catabolism. The chondroprotective effects of Abat inhibition on injury-induced OA implicate Abat, via its effects on cellular metabolism, as a promising pharmacological target for OA treatment.

\section{Methods}

Mice. Male 10-week-old C57BL/6J wild-type mice were purchased from The Jackson Laboratory (catalog 000664). MLI surgery was performed on the right knee as previously described (20). Sham surgery was performed on knees of the left limbs as a control. Lentivirus containing $10^{6}$ transducing units $(10 \mu \mathrm{l})$ was injected into the knee joint immediately after MLI to either knock down or overexpress Abat. Scramble lentivirus was used as a control. To inhibit Abat activity in vivo, vigabatrin $(50 \mathrm{mg} / \mathrm{kg}$ and $200 \mathrm{mg} / \mathrm{kg}$, Tocris, 0808) was administered by intraperitoneal injection to mice daily following MLI surgery for 6 or 10 weeks.

Murine knee joint analysis. Knee joint samples were harvested at different time points for histological and micro-CT analyses. Specifically, knee joints were harvested 4 weeks after MLI in Abat overexpression treat- 
ment groups, 10 weeks for Abat inhibition treatment, or 6 and 10 weeks for vigabatrin-treated mice. After neutral-buffered formalin fixation and EDTA decalcification, knee joints were processed, paraffin embedded, and sectioned at $5 \mu \mathrm{m}$. Safranin-O/Fast Green (Sigma, F7258) and Alcian Blue Hematoxylin/Orange G (Sigma, O7252) stainings were performed to analyze knee joint tissue architecture. OA severity was scored using the established OARSI scoring system (56). Immunohistochemistry was performed on sections using citrate buffer antigen retrieval and colorimetric development methodologies. Antibodies against Prg4 (1:200, Abcam, ab28484), Mmp13 (1:200, Abcam, ab39012), Col10A1 (1:100, Quartet, 1-CO097-05), FLAG (1:1000, Origene, TA150014), and GFP (1:200, Abcam, ab13970) were used. DAB reagent (Vector Laboratories, SK-4100) was used for peroxidase substrate reaction. Counterstaining was performed using hematoxylin or methyl green. Anti-GFP secondary antibody conjugated with Alexa Fluor 488 (1:100, Invitrogen, A21311) was used, and fluorescence was analyzed on a Zeiss microscope.

Micro-CT analyses were performed on mouse knee joints before decalcification using a VivaCT 40 scanner (Scanco USA Inc.), using a protocol consisting of high-resolution (10.5 microns) x-ray energy settings of $55 \mathrm{kVp}, 145 \mu \mathrm{A}$, and $300 \mathrm{~ms}$ integration time. BV/TV of tibial subchondral bone as well as trabecular properties, including trabecular thickness, trabecular spacing, and trabecular number, were analyzed as previously described using the Scanco analysis software (57). In addition to the whole tibial plateau, analyses were also conducted on the medial tibial plateau to reveal more apparent pathological changes attributable to MLI.

Primary articular chondrocyte isolation and culture. Primary articular chondrocytes were isolated from murine femoral heads and human cartilage as previously described (20). Briefly, cells were harvested from cartilage tissue by $4-6$ hours of collagenase $\mathrm{P}(0.5 \mathrm{mg} / \mathrm{ml}$, Roche, 11249002001$)$ digestion for mice and 14-16 hours of collagenase $\mathrm{P}(0.035 \%)$ digestion for human tissue. Following digestion, cells were seeded at a density of $5 \times 10^{5}, 2.5 \times 10^{5}$, or $5 \times 10^{4}$ cells/well in 12-, 24-, or 96-well plates, respectively. For the Dnmt3b LOF experiments, chondrocytes isolated from $D n m t 3 b^{f / f l}$ mice were transduced with adenovirus expressing GFP (control) or Cre (Dnmt3b LOF) at a multiplicity of 50. Lenti-shAbat-GFP (Origene, TL508059V) transduction at a multiplicity of 10 for 24 hours or vigabatrin $(100 \mu \mathrm{M})$ pretreatment for 30 minutes was used to knock down Abat expression or inhibit Abat activity, followed by IL-1 $\beta$ treatment (1 ng/ml, R\&D Systems, 201-LB-005) for 24 hours. Lenti-Dnmt3b (Origene, MR225598L1V) and lenti-AbatFlag (ABM, LVP457865) at a multiplicity of 5 were used to induce Dnmt3b and Abat overexpression in primary articular chondrocytes.

Full-thickness sections of human articular cartilage were removed from the femoral condyles and tibial plateaus of specimens obtained following total knee replacement surgery, diced into 2-mm cubes, and digested at $37^{\circ} \mathrm{C}$ for 90 minutes by $0.4 \%$ pronase (Roche, 10165921001 ) and 14 hours by $0.035 \%$ collagenase P (Roche, 11249002001). The tissue from the area of the joint surface with severe degeneration was used as OA samples, and tissue from the cartilage with normal appearance was used as nondiseased samples (20). Cells were plated at a density of 100,000 cells/ $\mathrm{cm}^{2}$ and treated with IL-1 $\beta(10 \mathrm{ng} / \mathrm{ml}$, Roche, 201-LB-005) for 48 hours.

Mitochondria stress test. Mitochondrial respiration profile, particularly OCR, was measured according to the manufacturer's instructions (Agilent). Briefly, primary articular chondrocytes with Abat overexpression via lenti-Abat as well as Abat inhibition by lenti-shAbat and vigabatrin were plated in XF96 plates at $5 \times 10^{4}$ cells/well with XF Assay Medium Modified DMEM (Seahorse, 101022-100). Oligomycin and FCCP were used at a final concentration of $5 \mu \mathrm{M}$ and $1 \mu \mathrm{M}$, respectively, to manipulate the mitochondrial response. Antimycin and rotenone (Agilent) were used to suppress the mitochondrial respiration. At the end of the assays, protein concentrations were measured for normalization.

ChIP assay. ATDC-5 cells $\left(1 \times 10^{7}\right)$ purchased from MilliporeSigma (catalog 99072806) were harvested and fixed with formaldehyde for ChIP assays. Chromatin was fragmented by sonication to shear DNA to $200-500 \mathrm{bp}$ in size. Chromatin fragments were immunoprecipitated using the Dnmt3b antibody (1:50, Abcam, ab2851) while Histone H3 and IgG antibodies (Cell Signaling, 56383) were used as positive and negative controls, respectively. After reverse cross-linking of protein DNA, the enrichment of Abat DNA fragments during immunoprecipitation was analyzed by standard PCR and quantitative real-time PCR. Primer 1 sequences (within $\mathrm{CpG}$ island) for Abat promoter used for PCR were forward, 5'-GGAAATCCCTCCCTCGTTTAG-3', and reverse, 5'-GGAAAGGGAGATGAGAAAG-3'; primer 2 sequences (away from CpG island) were forward, 5'-GCTAGCCGCTTAAACTCATCTA-3', and reverse, 5'-GAGATTGGAAAGGAGAAGG-3'.

GABA concentration measurement. Vigabatrin efficacy in vivo and in vitro was examined by the GABA 
Table 1. Primer sequences for qPCR

\begin{tabular}{|c|c|}
\hline Genes & Sequences \\
\hline Col10a1 & $\begin{array}{c}\text { 5'-ATCCCTTCTTCTCCTCTTACTG-3' } \\
\text { 5'-TGCTCAACGGTACCAAACG-3' }\end{array}$ \\
\hline Runx2 & $\begin{array}{c}\text { 5'-CGTCCACTGTCACTTTAAATAGCTC-3' } \\
\text { 5'-GTAGCCAGGTTCAACGATCTG-3' }\end{array}$ \\
\hline Mmp13 & $\begin{array}{l}\text { 5'-AGACTGGTAATGGCATCAAGG-3' } \\
\text { 5'-GCCATTTCATGCTTCCTCATG-3' }\end{array}$ \\
\hline Abat & $\begin{array}{c}\text { 5'-GGACTTCCGTCTTCATCACTG-3' } \\
\text { 5'-ACCTCCACCTCTTCATACCT-3' }\end{array}$ \\
\hline ACTB & $\begin{array}{l}\text { 5'-AGATGTGGATCAGCAAGCAG-3' } \\
\text { 5'-GCGCAAGTTAGGTTTTGTCA-3' }\end{array}$ \\
\hline$A B A T$ & $\begin{array}{l}\text { 5'-CAGGTGTTCAAGATCCGGTAG-3' } \\
\text { 5'-CAGCAGACGTGATCACCTTC-3' }\end{array}$ \\
\hline GAPDH & $\begin{array}{l}\text { 5'-TGTAGTTGAGGTCAATGAAGGG-3' } \\
\text { 5'-ACATCGCTCAGACACCATG-3' }\end{array}$ \\
\hline
\end{tabular}

ELISA Kit (LSBio, LS-F4121) using brain tissue isolated from vigabatrin-treated mice and cell lysates from vigabatrin-treated articular chondrocytes according to the manufacturer's instructions.

Methylation $q P C R$. Methylation qPCR for Abat was performed according to the manufacturer's instructions (Qiagen, 335452). Genomic DNA isolated from Dnmt3b LOF and control articular chondrocytes was enzymatically digested by methylation-sensitive or methylation-dependent enzymes separately. The enzyme-digested genomic DNA was then used for $\mathrm{qPCR}$, and the primers for Abat methylation (CpG island 104232) were obtained from Qiagen (EPMM104232).

Real-time PCR and Western blot. RNA was isolated from primary articular chondrocytes or murine cartilage tissue using the RNeasy Mini Kit (Qiagen, 74134). cDNA was synthesized using iScript kit (Bio-Rad, 1708841), and reverse transcription PCR was performed according to the manufacturer's instructions. Primer sequences for Runx2, Mmp13, Col10a1, Abat, Abatb, ABAT, and GAPDH are shown in Table 1. Western blot analyses were performed on protein lysates from either primary articular chondrocytes or murine cartilage tissue using the primary antibodies Abat (1:1000, LSBio, LS-B14889) and $\beta$-actin (1:4000, MilliporeSigma, 2228).

Statistics. All data are presented as the mean $\pm \mathrm{SD}$. The comparisons between 2 groups were analyzed by 2 -tailed Student's $t$ test. Multiple-factorial 1-way ANOVA was used to compare multiple groups followed by post hoc tests. $P<0.05$ was considered statistically significant.

Study approval. Human knee articular cartilage tissue was obtained from surgeons at Washington University Department of Orthopedic Surgery following total knee replacement surgery. All studies involving this tissue, which was used to generate primary articular chondrocytes for in vitro experiments, were approved by Washington University Human Research Protection Office (IRB ID 201104119). MLI procedures were conducted in compliance with the Association for Assessment and Accreditation of Laboratory Animal Care-accredited Washington University IACUC and the NIH guidelines.

\section{Author contributions}

JS, CW, JY, TX, and RJO conceived and designed experiments. JS, CW, JY and TX performed experiments. JS, CW, JY, TX, AM, and RJO analyzed and interpreted data. JS, CW, AM, and RJO wrote and edited the manuscript.

\section{Acknowledgments}

We would like to gratefully acknowledge the technical expertise and assistance from Daniel Leib and Michael Brodt for micro-CT scanning and analyses and Crystal Idleburg and Samantha Coleman (Department of Orthopaedic Surgery, School of Medicine, Washington University in St. Louis, St. Louis, Missouri, USA) for histology services. This work was supported by grants from NIH R01 AR069605 to RJO and P30 AR057235 to Washington University Musculoskeletal Research Center.

Address correspondence to: Regis J. O'Keefe, Department of Orthopaedic Surgery, Washington University 
in St. Louis, 660 South Euclid, CB 8233, St. Louis, Missouri 63110, USA. Phone: 314.747.8414; Email: rokeefe@wustl.edu.

1. van Baar ME, Dekker J, Lemmens JA, Oostendorp RA, Bijlsma JW. Pain and disability in patients with osteoarthritis of hip or knee: the relationship with articular, kinesiological, and psychological characteristics. J Rheumatol. 1998;25(1):125-133.

2. Woolf AD, Erwin J, March L. The need to address the burden of musculoskeletal conditions. Best Pract Res Clin Rheumatol. 2012;26(2):183-224

3. Buckwalter JA, Saltzman C, Brown T. The impact of osteoarthritis: implications for research. Clin Orthop Relat Res. 2004;(suppl 427):S6-15.

4. Cutler DM, Ghosh K. The potential for cost savings through bundled episode payments. N Engl J Med. 2012;366(12):1075-1077.

5. Hootman JM, Helmick CG. Projections of US prevalence of arthritis and associated activity limitations. Arthritis Rheum. 2006;54(1):226-229.

6. Yu SP, Hunter DJ. Emerging drugs for the treatment of knee osteoarthritis. Expert Opin Emerg Drugs. 2015;20(3):361-378.

7. Tonge DP, Pearson MJ, Jones SW. The hallmarks of osteoarthritis and the potential to develop personalised disease-modifying pharmacological therapeutics. Osteoarthr Cartil. 2014;22(5):609-621.

8. Goldring MB, Berenbaum F. Emerging targets in osteoarthritis therapy. Curr Opin Pharmacol. 2015;22:51-63.

9. Chen D, et al. Osteoarthritis: toward a comprehensive understanding of pathological mechanism. Bone Res. 2017;5:16044.

10. Valdes AM, et al. Genetic variation in the SMAD3 gene is associated with hip and knee osteoarthritis. Arthritis Rheum. 2010;62(8):2347-2352.

11. Valdes AM, et al. The GDF5 rs143383 polymorphism is associated with osteoarthritis of the knee with genome-wide statistical significance. Ann Rheum Dis. 2011;70(5):873-875.

12. Panoutsopoulou K, Zeggini E. Advances in osteoarthritis genetics. J Med Genet. 2013;50(11):715-724

13. Reynard LN, Loughlin J. Insights from human genetic studies into the pathways involved in osteoarthritis. Nat Rev Rheumatol. 2013;9(10):573-583

14. Evangelou E, et al. A meta-analysis of genome-wide association studies identifies novel variants associated with osteoarthritis of the hip. Ann Rheum Dis. 2014;73(12):2130-2136.

15. Rodriguez-Fontenla C, et al. Assessment of osteoarthritis candidate genes in a meta-analysis of nine genome-wide association studies. Arthritis Rheumatol. 2014;66(4):940-949.

16. Goldring MB, Marcu KB. Epigenomic and microRNA-mediated regulation in cartilage development, homeostasis, and osteoarthritis. Trends Mol Med. 2012;18(2):109-118.

17. Barter MJ, Bui C, Young DA. Epigenetic mechanisms in cartilage and osteoarthritis: DNA methylation, histone modifications and microRNAs. Osteoarthr Cartil. 2012;20(5):339-349.

18. Loughlin J, Reynard LN. Osteoarthritis: epigenetics of articular cartilage in knee and hip OA. Nat Rev Rheumatol. 2015;11(1):6-7.

19. Shen J, Abu-Amer Y, O'Keefe RJ, McAlinden A. Inflammation and epigenetic regulation in osteoarthritis. Connect Tissue Res. 2017;58(1):49-63.

20. Shen J, et al. DNA methyltransferase $3 \mathrm{~b}$ regulates articular cartilage homeostasis by altering metabolism. JCI Insight. 2017;2(12):93612

21. Mobasheri A, Rayman MP, Gualillo O, Sellam J, van der Kraan P, Fearon U. The role of metabolism in the pathogenesis of osteoarthritis. Nat Rev Rheumatol. 2017;13(5):302-311.

22. June RK, Liu-Bryan R, Long F, Griffin TM. Emerging role of metabolic signaling in synovial joint remodeling and osteoarthritis. J Orthop Res. 2016;34(12):2048-2058.

23. Rajpurohit R, Koch CJ, Tao Z, Teixeira CM, Shapiro IM. Adaptation of chondrocytes to low oxygen tension: relationship between hypoxia and cellular metabolism. J Cell Physiol. 1996;168(2):424-432.

24. Heywood HK, Knight MM, Lee DA. Both superficial and deep zone articular chondrocyte subpopulations exhibit the Crabtree effect but have different basal oxygen consumption rates. J Cell Physiol. 2010;223(3):630-639.

25. Wang C, Silverman RM, Shen J, O'Keefe RJ. Distinct metabolic programs induced by TGF- $\beta 1$ and BMP2 in human articular chondrocytes with osteoarthritis. J Orthop Translat. 2018;12:66-73.

26. Osei YD, Churchich JE. Screening and sequence determination of a cDNA encoding the human brain 4-aminobutyrate aminotransferase. Gene. 1995;155(2):185-187.

27. Besse A, et al. The GABA transaminase, ABAT, is essential for mitochondrial nucleoside metabolism. Cell Metab. 2015;21(3):417-427.

28. Jaeken J, et al. Gamma-aminobutyric acid-transaminase deficiency: a newly recognized inborn error of neurotransmitter metabolism. Neuropediatrics. 1984;15(3):165-169.

29. Tsuji M, et al. A new case of GABA transaminase deficiency facilitated by proton MR spectroscopy. J Inherit Metab Dis. 2010;33(1):85-90

30. Millan MJ, et al. Cognitive dysfunction in psychiatric disorders: characteristics, causes and the quest for improved therapy. Nat Rev Drug Discov. 2012;11(2):141-168.

31. Rogawski MA, Löscher W. The neurobiology of antiepileptic drugs. Nat Rev Neurosci. 2004;5(7):553-564.

32. Rudolph U, Möhler H. GABAA receptor subtypes: therapeutic potential in Down syndrome, affective disorders, schizophrenia, and autism. Annu Rev Pharmacol Toxicol. 2014;54:483-507.

33. Gao SG, et al. Association between Wnt inhibitory factor-1 expression levels in articular cartilage and the disease severity of patients with osteoarthritis of the knee. Exp Ther Med. 2016;11(4):1405-1409.

34. Sarup A, Larsson OM, Schousboe A. GABA transporters and GABA-transaminase as drug targets. Curr Drug Targets CNS Neurol Disord. 2003;2(4):269-277.

35. Grant SM, Heel RC. Vigabatrin. A review of its pharmacodynamic and pharmacokinetic properties, and therapeutic potential in epilepsy and disorders of motor control. Drugs. 1991;41(6):889-926. 
36. Ben-Menachem E. Mechanism of action of vigabatrin(192): correcting misperceptions. Acta Neurol Scand Suppl. 2011;(192):5-15.

37. Wong CG, Bottiglieri T, Snead OC. GABA, gamma-hydroxybutyric acid, and neurological disease. Ann Neurol. 2003;54(suppl 6):S3-12.

38. Pollesello P, de Bernard B, Grandolfo M, Paoletti S, Vittur F, Kvam BJ. Energy state of chondrocytes assessed by 31 P-NMR studies of preosseous cartilage. Biochem Biophys Res Commun. 1991;180(1):216-222.

39. Fernie AR, Carrari F, Sweetlove LJ. Respiratory metabolism: glycolysis, the TCA cycle and mitochondrial electron transport. Curr Opin Plant Biol. 2004;7(3):254-261.

40. Haegele KD, Schechter PJ. Kinetics of the enantiomers of vigabatrin after an oral dose of the racemate or the active S-enantiomer. Clin Pharmacol Ther. 1986;40(5):581-586.

41. Frisk-Holmberg M, Kerth P, Meyer P. Effect of food on the absorption of vigabatrin. Br J Clin Pharmacol. 1989;27(suppl 1):23S-25S.

42. Schechter PJ. Clinical pharmacology of vigabatrin. Br J Clin Pharmacol. 1989;27(suppl 1):19S-22S.

43. Richens A. Pharmacology and clinical pharmacology of vigabatrin. J Child Neurol. 1991;suppl 2:S7-S10.

44. Tannahill GM, et al. Succinate is an inflammatory signal that induces IL-1 $\beta$ through HIF-1 $\alpha$. Nature. 2013;496(7444):238-242.

45. Mills E, O’Neill LA. Succinate: a metabolic signal in inflammation. Trends Cell Biol. 2014;24(5):313-320.

46. Rubic T, et al. Triggering the succinate receptor GPR91 on dendritic cells enhances immunity. Nat Immunol. 2008;9(11):1261-1269.

47. Selak MA, et al. Succinate links TCA cycle dysfunction to oncogenesis by inhibiting HIF- $\alpha$ prolyl hydroxylase. Cancer Cell. 2005;7(1):77-85.

48. Pistollato F, et al. Hypoxia and succinate antagonize 2-deoxyglucose effects on glioblastoma. Biochem Pharmacol. 2010;80(10):1517-1527.

49. Li Y, et al. Succinate/NLRP3 inflammasome induces synovial fibroblast activation: therapeutical effects of clematichinenoside AR on arthritis. Front Immunol. 2016;7:532

50. Zhang B, McDaniel SS, Rensing NR, Wong M. Vigabatrin inhibits seizures and mTOR pathway activation in a mouse model of tuberous sclerosis complex. PLoS One. 2013;8(2):e57445.

51. Yan B, et al. mTORC1 regulates PTHrP to coordinate chondrocyte growth, proliferation and differentiation. Nat Commun. 2016;7:11151

52. Chen J, Long F. mTOR signaling in skeletal development and disease. Bone Res. 2018;6:1

53. Bartolomeo R, et al. mTORC1 hyperactivation arrests bone growth in lysosomal storage disorders by suppressing autophagy. J Clin Invest. 2017;127(10):3717-3729.

54. Jung CH, Ro SH, Cao J, Otto NM, Kim DH. mTOR regulation of autophagy. FEBS Lett. 2010;584(7):1287-1295.

55. Kim YC, Guan KL. mTOR: a pharmacologic target for autophagy regulation. J Clin Invest. 2015;125(1):25-32.

56. Glasson SS, Chambers MG, Van Den Berg WB, Little CB. The OARSI histopathology initiative - recommendations for histological assessments of osteoarthritis in the mouse. Osteoarthr Cartil. 2010;18(suppl 3):S17-S23.

57. Sampson ER, et al. Establishment of an index with increased sensitivity for assessing murine arthritis. J Orthop Res. 2011;29(8):1145-1151. 